\title{
Evolution and Self-renewal of the Journal of Mammary Gland Biology and Neoplasia
}

\author{
Russell C. Hovey ${ }^{1}$ (D) Z Zuzana Koledova ${ }^{2}$ (D)
}

Received: 14 September 2021 / Accepted: 16 September 2021 / Published online: 14 October 2021

(c) The Author(s), under exclusive licence to Springer Science+Business Media, LLC, part of Springer Nature 2021

Keywords Breast $\cdot$ Mammary gland $\cdot$ Milk $\cdot$ Artificial intelligence $\cdot$ Precision therapy

\section{Editorial}

The mammary gland is a fascinating and specialized organ that unites all mammals, having evolved more than 200 million years ago for its ability to provide a selective evolutionary advantage to females so as to ensure the survival of their progeny across a wide range of environments [1]. The range of specialized molecules in this nutrient-rich food also protects the infant from a range of pathogens to ensure their healthy growth and development. The Journal of Mammary Gland Biology and Neoplasia was created by human action 26 years ago. Yet, it has analogously evolved according to the needs of the scientific community and selective pressures of the funding and publishing climate. Over the years the Journal has stably provided a respected medium for disseminating the latest research in the areas of mammary gland development, its function, and disease, which in turn nurtures the next generation of mammary gland biologists and breast cancer researchers.

Similar to the mammary gland, which relies on selfrenewing mammary stem cells to build epithelial structures for efficient milk production, the Journal relies on its dedicated Editorial board to provide new ideas, healthy submission numbers through their networks, and efficient peer-review to sustain a successful publication. Like the endocrine system that coordinates many aspects of mammary gland function, Dr. Zuzana Koledova is taking the

Russell C. Hovey

rchovey@ucdavis.edu

$\triangle$ Zuzana Koledova

koledova@med.muni.cz

1 Department of Animal Science, University of California, Davis, CA, USA

2 Department of Histology and Embryology, Faculty of Medicine, Masaryk University, Brno, Czech Republic reins of the Journal to coordinate its activities as the new Editor-in-Chief. She replaces Prof. Russ Hovey, who has worked tirelessly for the last 11 years to sustain the quality of the Journal, to raise its profile and to adjust it to the current needs of the field. Under Prof. Hovey's leadership, the Journal underwent several important transformations. First, it evolved from a compendium of thematic issues containing only solicited reviews to a publication accepting both highquality peer-reviewed original research and review articles (both solicited and unsolicited) [2]. Second, the Journal of Mammary Gland Biology and Neoplasia became a transformative journal, offering the possibility for authors to publish Open Access. Furthermore, to connect with the next generation of mammary gland biologists and to support its trainees, Prof. Hovey initiated a Facebook page to promote the contents of the Journal and the greater research community (https://www.facebook.com/mammaryglandbiologists). The Journal also now has an annual student award for the best manuscript which, like a second parity, encourages a highly-productive and efficient next generation of mammary gland biologists.

Dr. Koledova is no stranger to the Journal's editorial team. As Associate Editor for the past year, she recently acted as a Guest Editor for two special issues of the journal, including Unraveling the Breast: Advances in Mammary Biology and Cancer Methods [3] and Connecting the Dots: Mammary Gland and Breast Cancer at Single Cell Resolution [4]. She also created the Journal's exciting new cover design, effective from December 2020, and has further increased Journal's presence on social media by starting the Journal's Twitter account (@Mammary_journal). In her new role as the Editor-in-Chief, she looks forward to continuing development of the Journal, introducing new article types, exploring a new title for the Journal, and embracing the open science movement, while maintaining the Journal's commitment to data integrity and to publishing high-quality science. 
Relying on the help and support of the Editorial board and our dedicated Managing Editor, Erika Ginsburg, she also braces herself to face challenges such as an increasing incidence of ethical misconduct, a decreasing availability of peer reviewers, and variable numbers of manuscript submissions as scientists face pressures from their funding agencies and institutions to publish in elite journals. Yet, overcoming all these challenges is necessary and worthwhile in order to sustain this important publication that stands as the one integrated source of information on development, pathology and function of the mammary gland, which also has a global social, economic and environmental impact.

Looking to the future, there are many reasons why mammary gland biologists and the critical research they undertake is more important than ever. For starters, the global dairy industry relies on dairy animals to provide milk for a diversity of foodstuffs for human nutrition, which are preserved and processed for consumption across the planet. At the same time, the livestock sectors rely on the mammary glands to raise the next generation - whether that be piglets, lambs, calves, or foals, of which billions are born each year. And lastly, milk as a foodstuff has undergone a renaissance in recent years, not only through efforts to increase the uptake of breastfeeding worldwide, but also to capture a range of bioactive molecules from unique or abundant milks, and to use these to benefit infant human nutrition.

There are almost certain changes on the horizon, where a changing climate continues to increase pressure on resources such as water and nutrients, which are all essential for conversion by animals into milk. The livestock sector has been successfully applying scientific knowledge, such as selective breeding, improved nutrition, and better management of animal physiology and regulation of mammary gland function to make dairy animals far more efficient in producing milk than ever before. Looking forward, the landscape also presents tremendous opportunity for a real impact from genetic engineering with the advent of widespread genome editing technologies that have been integrated into a range of species. "Low-carbon livestock" can help produce enough food to eliminate hunger and malnourishment while minimizing the overall output of greenhouse gases [5]. Likewise efforts are underway in a number of venues to synthesize all the components of milk without the animal [6]. What remains to be seen - with all questions remaining with the consumer - is how public acceptance steers the uptake of all these technologies.

A changing climate also threatens the potential survival of species by compromising the way the mammary glands can nurture the next generation. Like all species, wildlife must consume the nutrients they need to produce milk, yet they face growing challenges to cover sufficient distances to access sufficient nutrients, alongside limited access to adequate water. Species also face greater thermal stress which changes the dynamics of metabolic state in support of lactation. And, to top it all off, the environment is increasingly contaminated by various chemicals that can appear in milk, which can have potentially long-lasting effects not only on infant performance but also cancer risk.

For humans, breast cancer remains a major threat to the health of women, but also men. It is the most frequently diagnosed type of cancer in women in the United States (besides skin cancer) and the second most common cause of death from cancer in women after lung cancer [7]. Yet, thanks to major multidisciplinary efforts and scientific advances, which have enabled early detection of the disease accompanied by novel treatments, the number of women who have died of breast cancer has decreased by $41 \%$ from 1989 to 2018 [7].

Technological advances in imaging, including breast tomosynthesis (3D mammography) and the adoption of artificial intelligence (AI) for computer-aided detection, diagnosis and triaging of breast cancers present new opportunities for improved screening and early detection as well as prediction of treatment outcomes. However, the ongoing risk of overdiagnosis, false-positive results, and, in the case of AI, the host of associated ethical, legal and regulatory challenges, all require further assessment. As a case in point, to adopt $\mathrm{AI}$ in breast imaging, it will be essential to integrate technology with upskilled healthcare professionals and updated clinical guidelines, and to define AI performance thresholds and legal accountability of algorithms, as well as the healthcare professionals using them [8].

Defining the signaling mechanisms at play during mammary gland development and breast cancer initiation and progression has been a cornerstone for the rational design of optimal treatment combinations to effectively eradicate the heterogeneous disease of breast cancer. Development of precision (mechanism-based) and personalized (patientsuited) therapies has raised the hope for more efficacious and less toxic treatments. Efforts in this area have increasingly employed patient-derived xenograft models and patientderived organoid techniques $[6,9,10]$. Advances in singlecell RNA sequencing and spatial transcriptomics open new avenues to deep-dive into the heterogeneity of breast tumors and its implications for therapeutic approaches. Major developments also lie at the front of cancer immunotherapy. Chimeric antigen receptor (CAR)-T cell therapy, approved in 2017 by the Food and Drug Administration for the treatment of children with acute lymphoblastic leukemia, has emerged as a promising immunotherapeutic strategy to improve the survival rates of patients with solid tumors, which do not respond to traditional targeted therapies, such as triple negative breast cancer. Moreover, human clinical trials of breast cancer vaccines are underway and herald a new era of hope for eradicating the disease. 
The importance and power of scientific research in solving a massive healthcare crisis with wide-ranging global socioeconomic impact has been recently demonstrated during the COVID-19 pandemic. Rapid development of COVID-19 tests and then COVID-19 vaccines, together with other anti-pandemic measures, have helped return the lives of many to somewhat (almost) normal routines. Once again, this pandemic has shown us how capable, problemsolving and flexible humans are in the midst of a crisis. Despite the many challenges associated with mandatory work from home due to closed universities, institutions and laboratories, scientists and students made use of the time to finish data analysis or to write articles, all while overcoming the challenges of online classes and meetings. They even managed to organize international conferences online, including, among others, two conferences, on which we bring reports in this issue; the Twelfth Annual ENBDC Workshop: Methods in Mammary Gland Biology and Breast Cancer [11] and the First Buenos Aires Breast Cancer Symposium [12]. Despite these rays of enthusiasm, as a field we have mourned the loss of several giants in the field of mammary gland biology in the past year, including Gil Smith [13], Zena Werb [14, 15], and recently Peter Hartmann.

In this issue, we present new research on changes in immune cell types in aging breast [16], interaction of BRCA1-mutated breast cancer cells with adipose-derived stromal cells [17], and sensitivity of the mammary glands to dietary fatty acids exposure in utero and postnatally [18]. Reviews summarize current knowledge on the role of NOTCH signaling in mammary gland development and breast cancer [19], discuss safety of estetrol-based formulations of contraceptives and menopause hormone therapy for breast health [20], and provide an appraisal of the relationship between mechanical environment of breast tissue and breast cancer risk with the focus on proteoglycans [21]. We hope that the Journal of Mammary Gland Biology and Neoplasia will continue to serve its role as the critical platform for disseminating the latest experimental results, for sharing technical approaches and methods, and for the review and discussion of new findings and even provocative ideas in the area of mammary gland development, lactation, and disease. Until this date, we published 847 articles in the Journal. With your support we hope to achieve the commemorative $1000^{\text {th }}$ publication soon. Will it be yours?

\section{Declarations}

Ethics Approval Not applicable

Conflict of Interest The authors are members of the Editorial board of the Journal of Mammary Gland Biology and Neoplasia.

\section{References}

1. Blackburn DG. Evolutionary origins of the mammary gland. Mammal Rev. 1991;21:81-96.

2. Hovey RC, Salomon D. The Journal of Mammary Gland Biology and Neoplasia into the future - the potential of plasticity and pluripotency. J Mammary Gland Biol Neoplasia. 2015;20:1-3.

3. Monkkonen T, Traustadóttir GÁ, Koledova Z. Unraveling the Breast: Advances in Mammary Biology and Cancer Methods. J Mammary Gland Biol Neoplasia. 2020;25:233-6.

4. van Amerongen R, Kordon EC, Koledova Z. Connecting the Dots: Mammary Gland and Breast Cancer at Single Cell Resolution. J Mammary Gland Biol Neoplasia. 2021;26:1-2.

5. FAO (2019) Five practical actions towards low-carbon livestock. Rome. http://www.fao.org/3/ca7089en/ca7089en.pdf.

6. Sumbal J, Budkova Z, Traustadóttir GÁ, Koledova Z. Mammary Organoids and 3D Cell Cultures: Old Dogs with New Tricks. J Mammary Gland Biol Neoplasia [Internet]. 2020 [cited 2020 Nov 22]; Available from https://doi.org/10.1007/s10911-020-09468-x.

7. Breast Cancer - Statistics [Internet]. Cancer Net 2012 [cited 2021 Sep 1]. Available from: https://www.cancer.net/cancer-types/ breast-cancer/statistics.

8. Hickman SE, Baxter GC, Gilbert FJ. Adoption of artificial intelligence in breast imaging: evaluation, ethical constraints and limitations. Br J Cancer. 2021;125:15-22.

9. Kawaguchi T, Foster BA, Young J, Takabe K. Current Update of Patient-Derived Xenograft Model for Translational Breast Cancer Research. J Mammary Gland Biol Neoplasia. 2017;22:131-9.

10. Juppet Q, De Martino F, Marcandalli E, Weigert M, Burri O, Unser M, et al. Deep Learning Enables Individual Xenograft Cell Classification in Histological Images by Analysis of Contextual Features. J Mammary Gland Biol Neoplasia. 2021;26:101-12.

11. Charifou E, Traustadottir GA, Bentires-Alj M, Howard B, Van Keymeulen A. Twelfth Annual ENBDC Workshop: Methods in Mammary Gland Biology and Breast Cancer. J Mammary Gland Biol Neoplasia. 2021. https://doi.org/10.1007/ s10911-021-09498-z.

12. Kordon E, Lanari C, Mando P, Novaro V, Rossi M, Simian M. The BA-BCS 2021: an initial "trial" for integrating basic science and medical progress on breast cancer in a Latin-American country. J Mammary Gland Biol Neoplasia. 2021. https://doi.org/ 10.1007/s10911-021-09501-7.

13. Medina D. In Memoriam - Gilbert Howlett Smith 1938-2020. J Mammary Gland Biol Neoplasia. 2020;25:177.

14. Barcellos-Hoff MH, Weaver VM. In Memoriam - Zena Werb 1945-2020. J Mammary Gland Biol Neoplasia. 2020;25:179-80.

15. Hovey R. The Editorial Board J of MGB and N. May Their Lineages Live on - an Editorial Board Tribute to Zena Werb and Gil Smith. J Mammary Gland Biol Neoplasia. 2021;25:173-5.

16. Zirbes A, Joseph J, Lopez JC, Sayaman RW, Basam M, Seewaldt VL, et al. Changes in Immune Cell Types with Age in Breast are Consistent with a Decline in Immune Surveillance and Increased Immunosuppression. J Mammary Gland Biol Neoplasia. 2021. https://doi.org/10.1007/s10911-021-09495-2.

17. Plangger A, Haslik W, Rath B, Neumayer C, Hamilton G. Interactions of BRCA1-mutated Breast Cancer Cell Lines with Adiposederived Stromal Cells (ADSCs). J Mammary Gland Biol Neoplasia. 2021. https://doi.org/10.1007/s10911-021-09493-4.

18. Berryhill GE, Gloviczki JM, Trott JF, Kraft J, Lock AL, Hovey RC. In utero exposure to trans-10, cis-12 conjugated linoleic acid modifies postnatal development of the mammary gland and its hormone responsiveness. J Mammary Gland Biol Neoplasia. 2021. https://doi.org/10.1007/s10911-021-09499-y.

19. Chen W, Wei W, Yu L, Ye Z, Huang F, Zhang L, et al. Mammary Development and Breast Cancer: a Notch Perspective. J 
Mammary Gland Biol Neoplasia. 2021. https://doi.org/10.1007/ s10911-021-09496-1.

20. Gallez A, Dias Da Silva I, Wuidar V, Foidart J-M, Péqueux C. Estetrol and Mammary Gland: Friends or Foes?. J Mammary Gland Biol Neoplasia. 2021. https://doi.org/10.1007/ s10911-021-09497-0.

21. Reye G, Huang X, Haupt LM, Murphy RJ, Northey JJ, Thompson EW, et al. Mechanical Pressure Driving Proteoglycan Expression in Mammographic Density: a Self-perpetuating Cycle?. J Mammary Gland Biol Neoplasia. 2021. https://doi.org/10.1007/ s10911-021-09494-3.

Publisher's Note Springer Nature remains neutral with regard to jurisdictional claims in published maps and institutional affiliations. 